\title{
Mucin Secretion in Cystic Fibrosis: A Systematic Review
}

\author{
Yaron Niv ${ }^{a}$ Samuel B. Ho ${ }^{b}$ Theodor Rokkas ${ }^{c}$ \\ ${ }^{a}$ Ministry of Health, Jerusalem, Israel; bepartment of Clinical Research, MBRU College of Medicine, Dubai, UAE; \\ 'Department of Gastroenterology, Henry Durant Medical Center, Athens, Greece
}

\section{Keywords}

Cystic fibrosis · Mucin · MUC5AC · MUC5B

\begin{abstract}
Background: Mucus protects the epithelium against invaders and toxic materials. Sticky and thick mucus is characteristic of CF. Objective: The aim of this systematic review is to characterize the specific mucins secreted in the lung and intestinal tract of CF patients. Methods: A systematic literature search was conducted up to December 31, 2019. The following terms were used: "cystic fibrosis" AND "mucin." Casecontrol studies comparing mucin expression in CF patients to healthy controls were included. Results: We found 741 eligible studies, 694 studies were rejected because they were performed in animals and not in full text, and 32 studies were excluded being editorials, duplications, review articles, meta-analysis, or not in English. Fifteen studies were eligible for our study, including $150 \mathrm{CF}$ patients compared to 82 healthy controls, all fulfilled the inclusion criteria. The main mucin types expressed in the sinus submucosal glands, sputum, tracheobronchial surface epithelium, and lung submucosal glands were MUC5AC and MUC5B. Increase in the number of sinusoidal submucosal glands and expression of MUC5B was found in CF patients, but no such difference
\end{abstract}

from healthy controls was found for the number of goblet cells in the surface epithelium nor in the expression of MUC5AC. The opposite was found in the tracheobronchial surface epithelium and in the lungs. Conclusions: Increased expression of MUC5AC in the surface epithelium and of MUC5B in the subepithelial glands may be the result of higher secretion rate of mucin into the lumen of the respiratory tract, causing mucus plaque, infection, and inflammation.

๑) 2020 S. Karger AG, Basel

\section{Introduction}

Mucus layers cover epithelial surfaces in the respiratory and gastrointestinal tracts and protect the epithelium against invaders and toxic materials [1]. The main components of the mucus layer are mucins, high-molecular weight, heavily glycosylated O-linked glycoproteins, which are synthesized by the epithelium, and composed of 2 types: secreted and membrane bound [2]. More than 20 mucin genes exist in the human genome. Secretory mucins are secreted by goblet cells and contribute to a thin layer over the mucosal surfaces, while membranebound mucins are attached to the mucosa and also involved in cell signaling, immune modulation, cell motil- karger@karger.com

www.karger.com/ddi

Karger" (c) 2020 S. Karger AG, Base
Correspondence to:

Yaron Niv, yaron.niv@moh.gov.il 
ity, adhesion, and growth [2,3]. MUC5AC and MUC5B are the main mucins secreted in the lung, MUC5AC and MUC6 in the stomach, and MUC2 in the small intestine and the colon $[4,5]$. Change in mucin synthesis, secretion, or degradation may be a primary event or secondary to inflammation, infection, or carcinogenesis.

Cystic fibrosis (CF), a common genetic disease caused by mutations in the cystic fibrosis transmembrane conductance regulator (CFTR), is characterized by progressive lung disease and digestive organ pathology [6]. Sticky and thick mucus covering the epithelial surface of the bronchial tree, sinuses, pancreatic ducts, intestinal tract, and the reproductive tract is the main characteristic of $\mathrm{CF}$ [7]. Intestinal obstruction is often associated with CF, particularly the meconium ileus that affects $15 \%$ of $\mathrm{CF}$ newborns. Thick mucus blocks pancreatic ducts and results in pancreatic destruction during the second and third trimester of gestation.

CFTR regulates chloride secretion, the function of epithelial sodium channel, and bicarbonate transport, controlling the movement of water. Dehydration of the mucosal surfaces when CFTR is mutated results in changes of the mucin properties in all organs and tissues mentioned, leading to obstruction, infection, and inflammation with the signs and symptoms of the disease.

Analyzing MUC5AC and MUC6 in CF lung sputum revealed contradictory results. Henke et al. [6] demonstrated decreased concentration, while Kirkham et al. [7] found overproduction, especially in exacerbations. Similarly, the data on other organs' mucin secretion in CF are sparse and controversial. Montserrat et al. [8] demonstrated high mucin secretion from normal pancreatic cells provoked by extracellular ATP, but not from CF pancreatic cells. In both, ATP induced a rapid intracellular calcium mobilization. Thus, CFTR seemed to mediate ATP-dependent mucin secretion.

The aim of this systematic review is to characterize the specific mucins secreted in the lungs and intestinal tract of CF patients. Understanding better the pathogenesis of CF may facilitate development of new therapies, elongate survival, or prevent organ transplantation.

\section{Methods}

\section{Literature Search}

A systematic literature search was conducted of English language publications in MEDLINE, PubMed, Scopus, EMBASE, and CENTRAL, up to December 31, 2019. The following terms were used: "cystic fibrosis" AND "mucin." Relevant studies were screened according to established protocol. In addition, the refer-

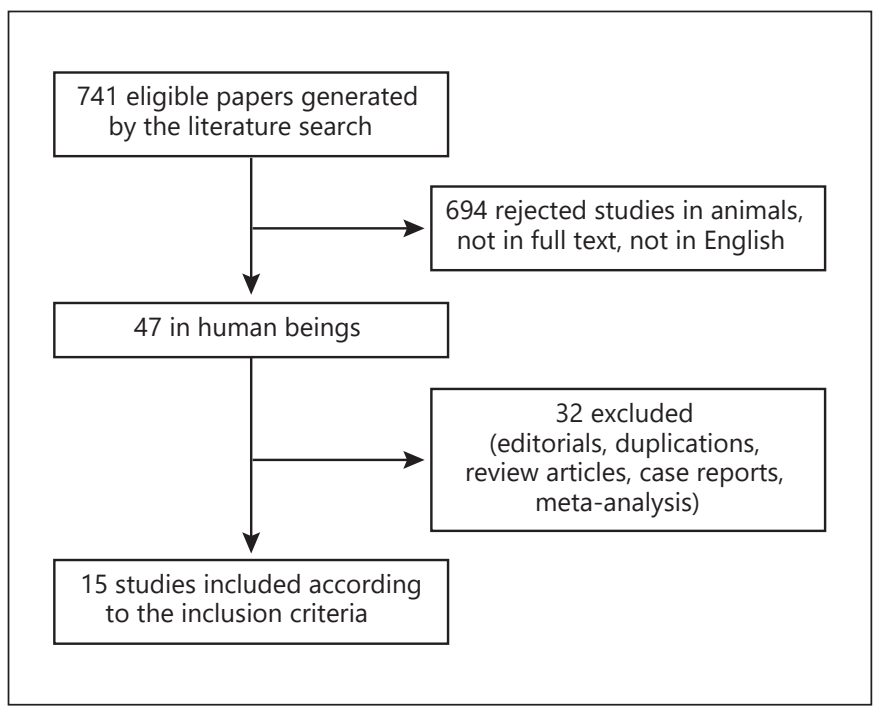

Fig. 1. Studies selected to the systematic review. Mucin secretion in CF. CF, cystic fibrosis.

ences of reviews were screened, and studies added when appropriate (Fig. 1).

\section{Inclusion and Exclusion Criteria - Study Selection and Data} Extraction

Case-control studies comparing mucin expression in CF patients to healthy controls were included. Mucin expression in the nasal mucosal surface, tracheobronchial mucosa, intestinal tract epithelium, pancreatic ducts mucosa, submucosal glands of the lung and sinuses, and the sputum was studied. Mucin expression studies included several different physical and chemical methods: purification by gel filtration chromatography, cesium chloride analytical gradient ultracentrifugation, protease treatment and disulfide bond reduction, in situ hybridization and immunocytochemistry for different MUCs mRNA and protein, mRNA slotblot (Northern blot), ELISA, Western blot after agarose gel electrophoresis, beta-elimination of O-linked oligosaccharides, and analysis by single-ion chromatography, comparing glycosylation.

\section{Results}

We found 741 eligible studies, 694 studies were rejected because they were performed in animals and not in full text, and 32 studies were excluded being editorials, duplications, review articles, meta-analysis, or not in English. Fifteen studies were found eligible, including $150 \mathrm{CF}$ patients compared to 82 healthy controls from 5 countries (USA, Canada, Germany, Sweden, and UK) that fulfilled the inclusion criteria, published till December 31, 2019 (Fig. 1; Table 1) [9-23]. 
Table 1. Mucin secretion in CF



CF, cystic fibrosis. 


\section{Study Description}

Wesley et al. [9] studied human intestinal mucins from 6 patients with CF and 8 controls which were prepared from tissue obtained at surgery and postmortem. Cesium chloride analytical gradient ultracentrifugation and carbohydrate side chains were examined. CF intestinal mucin was denser and more highly glycosylated than of controls and contained more sulfate. The increase in glycosylation resulted from a rise in fucose, galactose, and $\mathrm{N}$-acetylglucosamine.

Rose et al. [10] studied tracheobronchial mucins from healthy individuals and from patients with CF, which were isolated from lung mucus, purified, and their chemical and physical properties compared. Normal mucins required a dissociating and a reducing agent for solubilization. CF mucin was smaller than normal mucin due to intensive degradation.

Gupta and Jentoft [11] collected tracheobronchial mucin samples from controls and CF patients and purified them by gel filtration chromatography and by densitygradient centrifugation. Protease treatment and disulfide bond reduction were performed. Normal secretions contained higher molecular weight mucins than CF secretions. There was no change between $\mathrm{CF}$ patients and controls after protease treatment and disulfide bond reduction, and the same subunits of 2,000 and $300 \mathrm{kDa}$ were released. Thus, the main difference between CF patients and controls was a more effective process of degradation of mucin in the CF patients.

Li et al. [12] found that the MUC2 gene is expressed at 3- to 4-fold higher levels in CF nasal mucosa than in non-CF nasal mucosa and submucosal mucus-secreting glands. Reid et al. [13] demonstrated MUC6 expression in the material obstructing pancreatic duct of CF patients. They looked at mRNA (in situ hybridization) and the protein (immunocytochemistry). They also found similar distribution of MUC6 and CFTR in the pancreatic tissue.

Harris and Reid [14] could not demonstrate any change in MUC6 tandem repeat length between CF patients and controls. Voynow et al. [15] found a significant decrease in MUC5AC expression, using mRNA slot-blot (Northern blot), in the nasal mucosal epithelial cells of CF patients versus controls. No difference was demonstrated in the expression of MUC1 and MUC2.

Davies et al. [16] found high concentration of MUC5AC and MUC5B in the sputum of 5 CF patients, using immunochemistry and density-gradient centrifugation in $\mathrm{CsCl}$. In the sol phase, also small molecules that were the result of MUC5AC degradation were found. MUC2 was as a component of the insoluble residue from the gel, which accounted for $<4 \%$ by mass of the total mucins.

Groneberg et al. [17] found similar immunohistological staining to MUC5AC and MUCB in CF patients and healthy control, but hyperplasia of surface epithelial goblet cells was positive for MUC5AC in CF patients' trachea and bronchi. Kirkham et al. [18] found increased expression of MUC5B in CF and COPD patients in comparison with healthy controls. They used Western blot of sputum after agarose gel electrophoresis.

Schulz et al. [19] could not find any difference in glycosylation of MUC5AC or MUCB in the submucosal glands of airways in CF patients when compared with other lung diseases or healthy controls. They used betaelimination of $\mathrm{O}$-linked oligosaccharides and analysis by single-ion chromatography.

Henke et al. [20] compared MUC5AC and MUC5B protein expression in $11 \mathrm{CF}$ patients with lung disease remission and exacerbation and 12 healthy controls. A decrease of $89 \%$ in MUC5AC and of $40 \%$ in MUC5B was found in active $\mathrm{CF}$ patients in comparison with controls. An increase was demonstrated in exacerbation of the disease.

Guo et al. [21] found a significant association between MUC5AC variable number tandem repeat (VNTR) length and CF severity of lung involvement. Wu et al. [22] found a significant hyperplasia of submucosal glands of sinus mucosa in CF children, with an increased expression of MUC5B compared to normal controls. No change was demonstrated for goblet cells neither for MUC5AC expression.

Henderson et al. [23] were the first to demonstrate increase in mucin concentration of CF lung secretions using size exclusion chromatography and differential refractory techniques. They found 3-fold increase in MUC5B gene expression (measuring RNA) and 4.5- and 2.6-fold increases in MUC5AC and in MUC5B concentrations, respectively. Their findings contradicted those depended on immunochemistry which demonstrated the opposite. The explanation for the discrepancy may be that proteolytic degradation of the mucin backbone by specific enzymes caused disappearing of some antigenic sites. Increased mucin concentration caused increased osmotic pressure in the mucin layer, stasis, defective cilia, and cough-mediated mechanical clearance of bacteria, infection, and inflammation. The main mucin types expressed in the sinus submucosal glands, sputum, tracheobronchial surface epithelium, and lung submucosal glands were MUC5AC and MUC5B [16, 18, 22, 23] (Table 2).

Increase in the number of sinusoidal submucosal glands and in the expression of MUC5B was found in CF patients, but no such difference from healthy controls was found for 
Table 2. Mucin characteristics in CF

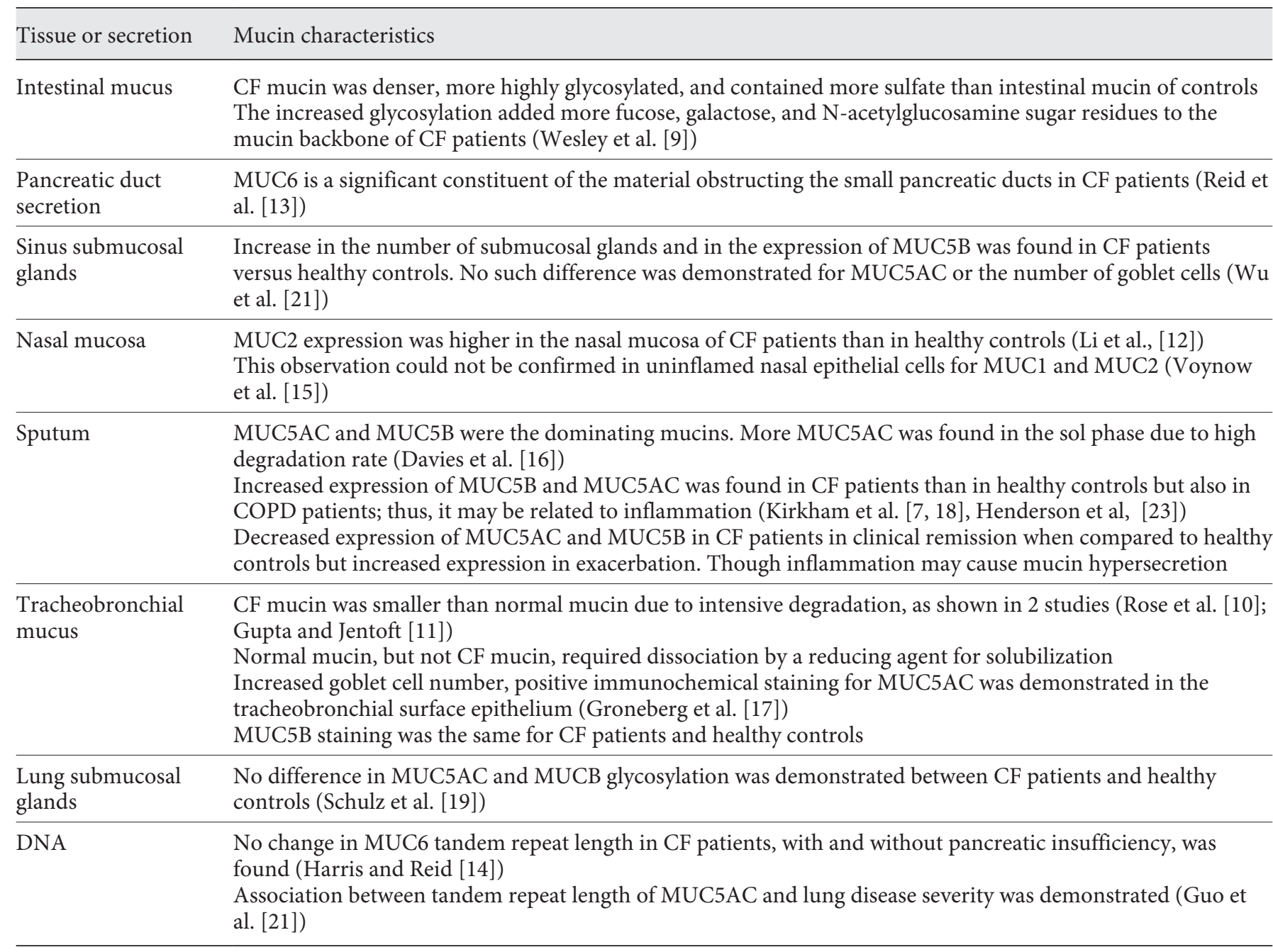

CF, cystic fibrosis.

the number of goblet cells in the surface epithelium nor in the expression of MUC5AC [22]. The opposite was found in the tracheobronchial surface epithelium and in the lungs, increased goblet cell number with a positive immunochemical staining for MUC5AC [17]. No such increase was found in the lung submucosal glands or for MUC5B expression [19]. Since increased expression of MUC5AC and MUC5B was also demonstrated in sputum of COPD patients, this might be the result of inflammation $[18,23]$. CF mucin of the tracheobronchial secretion was smaller than normal mucin due to intensive degradation, as shown in 2 studies $[10,11]$, in contrast with CF intestinal mucin which was denser, more highly glycosylated, and contained more sulfate than intestinal mucin of healthy controls [9].

Mucin Secretion in Cystic Fibrosis

\section{Discussion}

Although the pathophysiology of CF is related to the viscous and sticky mucus secreted by the epithelial surfaces of the respiratory and intestinal tracts, the interrelationship with nonfunctional CFTR is still unknown. Recently, it was found that CFTR is also able to transport bicarbonate, which is more linked to disease severity than the chloride secretion [24]. The reason for the lack of understanding the relation between CFTR and changes in mucin synthesis and secretion is the difficulty in studying the mucins. The main types of mucins involved in the etiopathogenesis of CF are gel-forming mucins MUC5B (synthesized in the submucosal glands) and MUC5AC 
(synthesized from the surface goblet cells) [25]. These mucins are heavily glycosylated proteins that occur as polymers and have a long backbone, or core protein, and many O-linked carbohydrate side chains. VNTR characterizes mucin, composed of different numbers of amino acids (typical for every mucin) and rich in amino acids serine, threonine, and proline. Proteases have difficulties to cut the protein backbone at this area of VNTR, and the mucin is relatively difficult to degrade. Mucin units are attached by a sulfide bond to form a huge viscoelastic molecule. This enormous size protein with the dense glycosylation poses methodological difficulties to study and characterization.

Assessment of mucin expression is done by several physical and chemical methods, such as purification by gel filtration chromatography on Sepharose 4B or 2B, cesium chloride analytical gradient ultracentrifugation and carbohydrate side chain examination, protease treatment and disulfide bond reduction, in situ hybridization and immunocytochemistry for different MUCs mRNA and protein, mRNA slot-blot (Northern blot), ELISA, Western blot after agarose gel electrophoresis, beta-elimination of O-linked oligosaccharides and analysis by singleion chromatography, comparing glycosylation, Southern blot, and PCR measurement of MUCs [26]. This richness of methods to measure mucin RNA or protein expression makes interpretation of many studies difficult and sometimes comparing results of studies which used different methods impossible.

Most of the studies found increased mucin expression, secretion, and concentration, followed by reactive increased enzymatic degradation $[9-11,15,23]$ and elongation of VNTR that correlated with disease severity [21]. Wesley et al. [9] described increased mucin density and more sulfate residues - these may explain the higher density caused by creation of more disulfide bridges between the heavy molecular mucin subunits. Thus, we speculate that all these changes make the mucin of CF patients extremely viscous, increase stasis, form mucus plagues, and prevent cilia function and clearance of bacteria. In addition, other mucins than MUC5AC and MUC5B, such as MUC2 [12] and MUC6 [13], are overexpressed in the pancreatic tissue, with similar distribution as CFTR [13] which may explain pancreatic duct pathology.

The role of microbiome in CF etiopathogenesis is unknown. One approach used capillary tubes instilled with artificial sputum intended to mimic CF physiologic conditions, which were then inoculated with bacterial strains derived from patients with CF collected during periods of clinical stability and exacerbation [27]. The investigators observed increased gas production and a 2 -unit reduction in $\mathrm{pH}$ before the onset of exacerbation. Theoretically, decrease in sputum $\mathrm{pH}$ may significantly increase mucin density.

Colorectal cancer incidence is higher in CF patients [28]. CFTR deficiency causes the mucus layers to become dehydrated and dysfunctional and permits bacterial contact with the epithelia. The inflammation may promote sequence of events that cause stem cell proliferation and colorectal cancer [28].

The results of the studies in our systematic review incline towards an important role for mucins in the etiopathogenesis of CF. Increased expression of MUC5AC in the surface epithelium and of MUC5B in the subepithelial glands may contribute to higher secretion rate of mucin into the lumen of the respiratory tract, causing mucus plaque, infection, and inflammation. Further studies should be conducted to explore the possible genetic or epigenetic changes in the mucin genes or the connection between CFTR mutation and mucin changes. A new era of management and treatment of CF will start with these studies, with new therapeutic options that will prevent lung transplantation or mortality.

\section{Statement of Ethics}

The research complies with the guidelines for human studies and was conducted ethically in accordance with the World Medical Association Declaration of Helsinki.

\section{Conflict of Interest Statement}

The authors have no conflicts of interest to declare.

\section{Funding Sources}

The authors did not receive any funding.

\section{Author Contributions}

Yaron Niv: acquisition of data, analysis and interpretation of data, and drafting of the manuscript. Samuel B. Ho: acquisition of data, analysis, and interpretation of data. Theodor Rokkas: study concept and design, analysis and interpretation of data, and drafting of the manuscript. 


\section{References}

1 Senapati S, Das S, Batra SK. Mucin-interacting proteins: from function to therapeutics. Trends Biochem Sci. 2010;35(4):236-45.

2 Corfield AP, Myerscough N, Longman R, Sylvester P, Arul S, Pignatelli M. Mucins and mucosal protection in the gastrointestinal tract: new prospects for mucins in the pathology of gastrointestinal disease. Gut. 2000;47(4):58994.

3 Sarosiek J, McCallum RW. What is the secretory potential of submucosal mucous glands within the human gullet in health and disease? Digestion. 1995;56(Suppl 1):15-23.

4 Kreda SM, Davis CW, Rose MC. CFTR, mucins, and mucus obstruction in cystic fibrosis. Cold Spring Harb Perspect Med. 2012;2(9): a009589-32.

5 Ehre C, Ridley C, Thornton DJ. Cystic fibrosis: an inherited disease affecting mucin-producing organs. Int J Biochem Cell Biol. 2014 52:136-45.

6 Henke MO, Renner A, Huber RM, Seeds MC, Rubin BK. MUC5AC and MUC5B mucins are decreased in cystic fibrosis airway secretions. Am J Respir Cell Mol Biol. 2004;31(1): 86-91.

7 Kirkham S, Sheehan JK, Knight D, Richardson PS, Thornton DJ. Heterogeneity of airways mucus: variations in the amounts and glycoforms of the major oligomeric mucins MUC5AC and MUC5B. Biochem J. 2002; 361(Pt 3):537-46.

8 Montserrat C, Merten M, Figarella C. Defective ATP-dependent mucin secretion by cystic fibrosis pancreatic epithelial cells. FEBS Lett. 1996;393(2-3):264-8.

9 Wesley A, Forstner J, Qureshi R, Mantle M, Forstner G. Human intestinal mucin in cystic fibrosis. Pediatr Res. 1983;17(1):65-9.

10 Rose MC, Brown CF, Jacoby JZ, Lynn WS, Kaufman B. Biochemical properties of tracheobronchial mucins from cystic fibrosis and non-cystic fibrosis individuals. Pediatr Res. 1987;22(5):545-51.
11 Gupta R, Jentoft N. The structure of tracheobronchial mucins from cystic fibrosis and control patients. J Biol Chem. 1992;267(5): 3160-7.

12 Li D, Wang D, Majumdar S, Jany B, Durham $\mathrm{SR}$, Cottrell J, et al. Localization and up-regulation of mucin (MUC2) gene expression in human nasal biopsies of patients with cystic fibrosis. J Pathol. 1997;181(3):305-10.

13 Reid CJ, Hyde K, Ho SB, Harris A. Cystic fibrosis of the pancreas: involvement of MUC6 mucin in obstruction of pancreatic ducts. Mol Med. 1997;3(6):403-11.

14 Harris A, Reid C. Cystic fibrosis and mucins. J Med Genet. 1998;35(1):82-3.

15 Voynow JA, Selby DM, Rose MC. Mucin gene expression (MUC1, MUC2, and MUC5/5AC) in nasal epithelial cells of cystic fibrosis, allergic rhinitis, and normal controls. Lung. 1998; 176:345-54.

16 Davies JR, Svitacheva N, Lannefors L, Kornfält R, Carlstedt I. Identification of MUC5B, MUC5AC and small amounts of MUC2 mucins in cystic fibrosis airway secretions. Biochem J. 1999;344(Pt 2):321-30.

17 Groneberg DA, Eynott PR, Oates T, Lim S, Wu R, Carlstedt I, et al. Expression of MUC5AC and MUC5B mucins in normal and cystic fibrosis lung. Respir Med. 2002; 96(2):81-6

18 Kirkham S, Sheehan JK, Knight D, Richardson PS, Thornton DJ. Heterogeneity of airways mucus: variations in the amounts and glycoforms of the major oligomeric mucins MUC5AC and MUC5B. Biochem J. 2002; 361(Pt 3):537-46.

19 Schulz BL, Sloane AJ, Robinson LJ, Sebastian LT, Glanville AR, Song Y, et al. Mucin glycosylation changes in cystic fibrosis lung disease are not manifest in submucosal gland secretions. Biochem J. 2005;387(Pt 3):911-9.
20 Henke MO, John G, Germann M, Lindemann $\mathrm{H}$, Rubin BK. MUC5AC and MUC5B mucins increase in cystic fibrosis airway secretions during pulmonary exacerbation. Am J Respir Crit Care Med. 2007;175(8):816-21.

21 Guo X, Pace RG, Stonebraker JR, Commander CW, Dang AT, Drumm ML, et al. Mucin variable number tandem repeat polymorphisms and severity of cystic fibrosis lung disease: significant association with MUC5AC. PLoS One. 2011;6(10):e25452.

22 Wu X, Amorn MM, Aujla PK, Rice S, Mimms $\mathrm{R}$, Watson AM, et al. Histologic characteristics and mucin immunohistochemistry of cystic fibrosis sinus mucosa. Arch Otolaryngol Head Neck Surg. 2011;137(4):383-9.

23 Henderson AG, Ehre C, Button B, Abdullah LH, Cai LH, Leigh MW, et al. Cystic fibrosis airway secretions exhibit mucin hyperconcentration and increased osmotic pressure. J Clin Invest. 2014;124(7):3047-60.

24 Garcia MA, Yang N, Quinton PM. Normal mouse intestinal mucus release requires cystic fibrosis transmembrane regulator-dependent bicarbonate secretion. J Clin Invest. 2009; 119(9):2613-22.

25 Caramori G, Di Gregorio C, Carlstedt I, Casolari P, Guzzinati I, Adcock IM, et al. Mucin expression in peripheral airways of patients with chronic obstructive pulmonary disease. Histopathology. 2004;45(5):477-84

26 Thomsson KA, Hansson GC, Kunzelman K. Identification and quantification of mucin expression. In: Amaral MD, Kunzelman K, editors. Cystic fibrosis, methods in molecular biology; 2011. p. 127-41

27 Yvonne JH, LiPuma JT. The microbiome in cystic fibrosis. Clin Chest Med. 2016;37:5967.

28 Scott P, Anderson K, Singhania M, Cormier R. Cystic fibrosis, CFTR, and colorectal cancer. Int J Mol Sci. 2020;21(8):2891-21. 